\title{
All-Optical 9.35 Gb/s Wavelength Conversion in an InP Photonic Crystal Nanocavity
}

\author{
Vukovic, Dragana; Yu, Yi; Heuck, Mikkel; Ek, Sara; Kuznetsova, Nadezda; Colman, Pierre; Palushani, \\ Evarist; Xu, Jing; Yvind, Kresten; Oxenløwe, Leif Katsuo
}

Total number of authors:

12

Published in:

ACP/IPOC 2013

Publication date:

2013

Document Version

Publisher's PDF, also known as Version of record

Link back to DTU Orbit

Citation (APA):

Vukovic, D., Yu, Y., Heuck, M., Ek, S., Kuznetsova, N., Colman, P., Palushani, E., Xu, J., Yvind, K., Oxenløwe, L. K., Mørk, J., \& Peucheret, C. (2013). All-Optical $9.35 \mathrm{~Gb} / \mathrm{s}$ Wavelength Conversion in an InP Photonic Crystal Nanocavity. In ACP/IPOC 2013 [AW4A.7] Optical Society of America.

\section{General rights}

Copyright and moral rights for the publications made accessible in the public portal are retained by the authors and/or other copyright owners and it is a condition of accessing publications that users recognise and abide by the legal requirements associated with these rights.

- Users may download and print one copy of any publication from the public portal for the purpose of private study or research.

- You may not further distribute the material or use it for any profit-making activity or commercial gain

- You may freely distribute the URL identifying the publication in the public portal 


\title{
All-Optical 9.35 Gb/s Wavelength Conversion in an InP Photonic Crystal Nanocavity
}

\author{
Dragana Vukovic, Yi Yu, Mikkel Heuck, Sara Ek, Nadezda Kuznetsova, Pierre Colman, \\ Evarist Palushani, Jing Xu, Kresten Yvind, Leif Katsuo Oxenløwe, Jesper Mørk, \\ and Christophe Peucheret \\ Department of Photonics Engineering, Technical University of Denmark, DK-2800 Kgs. Lyngby, Denmark \\ drvu@fotonik.dtu.dk
}

\begin{abstract}
Wavelength conversion of a $9.35 \mathrm{~Gb} / \mathrm{s} \mathrm{RZ}$ signal is demonstrated using an InP photonic crystal H0 nanocavity. A clear eye is observed for the converted signal showing a pre-FEC bit error ratio down to $10^{-3}$.

OCIS codes: (130.4815) Optical switching devices; (130.5296) Photonic crystal waveguides; (350.4238) Nanophotonics and photonic crystals.
\end{abstract}

\section{Introduction}

Photonic crystal $(\mathrm{PhC})$ cavities hold the promise of compact and low energy switching owing to the enhancement of nonlinearities resulting from their high quality $(Q$-) factor and reduced mode volume [1]. In particular, subfemtojoule switching energies have been demonstrated in InGaAsP H0 cavities close to the material bandgap edge, with fast carrier relaxation by exploiting fast carrier diffusion.

Even though the ultimate vision is to use $\mathrm{PhC}$ switches as building blocks in future telecommunication and data communication devices, it is interesting to notice that, with the notable exception of [2], no switching demonstrations have been performed so far with high bit rate modulated signals and detection techniques compatible with standard optical communication technology. Instead, switching results have been typically reported using pump-probe measurements with lock-in detection or waveform averaging with periodic signals [1,3]. In [2], $10 \mathrm{~Gb} / \mathrm{s}$ wavelength conversion was reported with a switching energy of $300 \mathrm{fJ}$, however using a relatively complex structure relying on hybrid integration of a III-V PhC nanocavity on a silicon-on-insulator platform. Being able to use simpler $\mathrm{PhC}$ devices within the transparency range of the material would be of high practical relevance.

In this paper we demonstrate wavelength conversion of a $10 \mathrm{~Gb} / \mathrm{s}$ return-to-zero (RZ) on-off keying (OOK) signal $(9.35 \mathrm{~Gb} / \mathrm{s}$ net rate) in a simple InP PhC switch with a single $\mathrm{H} 0$ cavity. The converted signal is detected using a conventional direct-detection receiver. To the best of our knowledge this is the first demonstration of wavelength conversion of telecom-grade signals in such a simple device.

\section{Sample description and experimental setup}

The structure used in the experiment consists of a single-mode $\mathrm{H} 0$ cavity coupled to two slab $\mathrm{PhC}$ waveguides on an InP membrane. A scanning electron microscope (SEM) image of the fabricated device is shown as an inset in Fig. 1. The $\mathrm{PhC}$ lattice constant, hole radius and membrane thickness are $420 \mathrm{~nm}, 92 \mathrm{~nm}$ and $340 \mathrm{~nm}$, respectively. The cavity is formed by shifting two neighboring air holes $84 \mathrm{~nm}$ in opposite directions resulting in ultra-small mode volume. The cavity is coupled with an input and output waveguide in the $\Gamma-\mathrm{K}$ direction. The waveguides are normal standard W1 defect waveguides but with the innermost row of holes adjacent to the waveguide shifted towards the center by $42 \mathrm{~nm}$. The coupling loss between lensed single mode fibers and the waveguide is estimated to be about $7.5 \mathrm{~dB}$ per facet. The detailed fabrication process is described in [4].

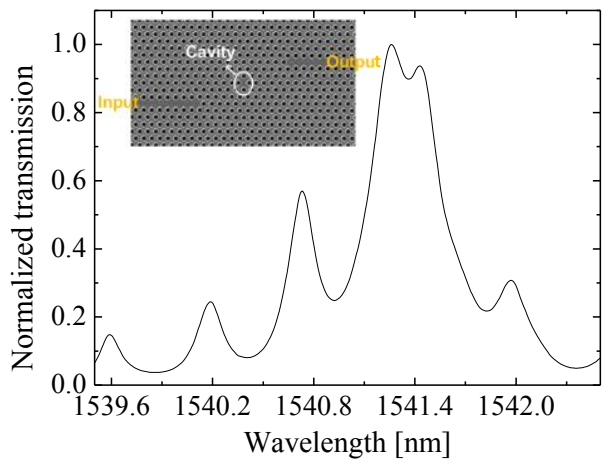

Fig. 1. Transmission spectrum of the cavity. Inset: SEM image of the fabricated sample. 


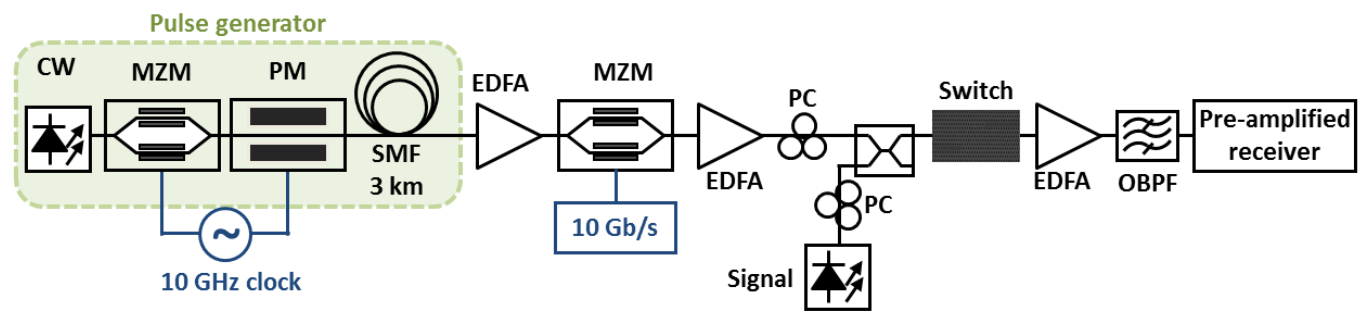

Fig. 2. Experimental setup for wavelength conversion.

Fig. 1 shows the cavity transmission spectrum measured using a wavelength tunable continuous wave (CW) laser. The cavity, designed to have Lorentzian line shape, has its resonance at $1541.33 \mathrm{~nm}$ and a loaded $Q$ - factor of 2700 . Increasing the power of a pump signal coupled to the cavity will shift the resonance towards shorter wavelengths due to a decrease of the refractive index induced by free carriers generated through two-photon absorption (TPA). This shift will in turn allow the transmission of a CW probe tuned to the blue side of the resonance, thus converting the intensity modulation of the pump to the probe wavelength, provided the carrier relaxation time is fast enough and the cavity linewidth is sufficiently large with respect to the modulation speed of the pump. Similar devices have been tested earlier in pump-probe measurements showing that about $8 \mathrm{~dB}$ of switching contrast could be achieved [5].

The wavelength conversion setup is shown in Fig. 2. The wavelength- and pulse-width tunable pump is generated starting from light emitted by a CW laser at $1540.95 \mathrm{~nm}$, which is intensity modulated in a Mach-Zehnder modulator (MZM) driven by a $10 \mathrm{GHz}$ radio frequency (RF) signal, resulting in $50 \% \mathrm{RZ}$ pulses. The pulses are then sinusoidally chirped in a phase modulator (PM) driven with a modulation index of 1.4 , and finally compressed in $3 \mathrm{~km}$ of standard single mode fiber (SMF), resulting in $15 \mathrm{ps}$ full-width at half maximum pulses at a repetition rate of $10 \mathrm{GHz}$. The pump is further intensity modulated at $10 \mathrm{~Gb} / \mathrm{s}$ in another MZM with a $2^{7}-1$ pseudo-random binary sequence (PRBS). A CW signal at $1540.33 \mathrm{~nm}$ is coupled together with the modulated pump in the PhC device with $4 \mathrm{dBm}$ and $6.8 \mathrm{dBm}$ average power, respectively. The states of polarization of both pump and signal are aligned to the TE mode of the input waveguide. At the output of the device, the combined signal is first amplified before the converted signal is selected with an optical band pass filter (OBPF) and input to a conventional pre-amplified receiver for bit error ratio (BER) measurements.
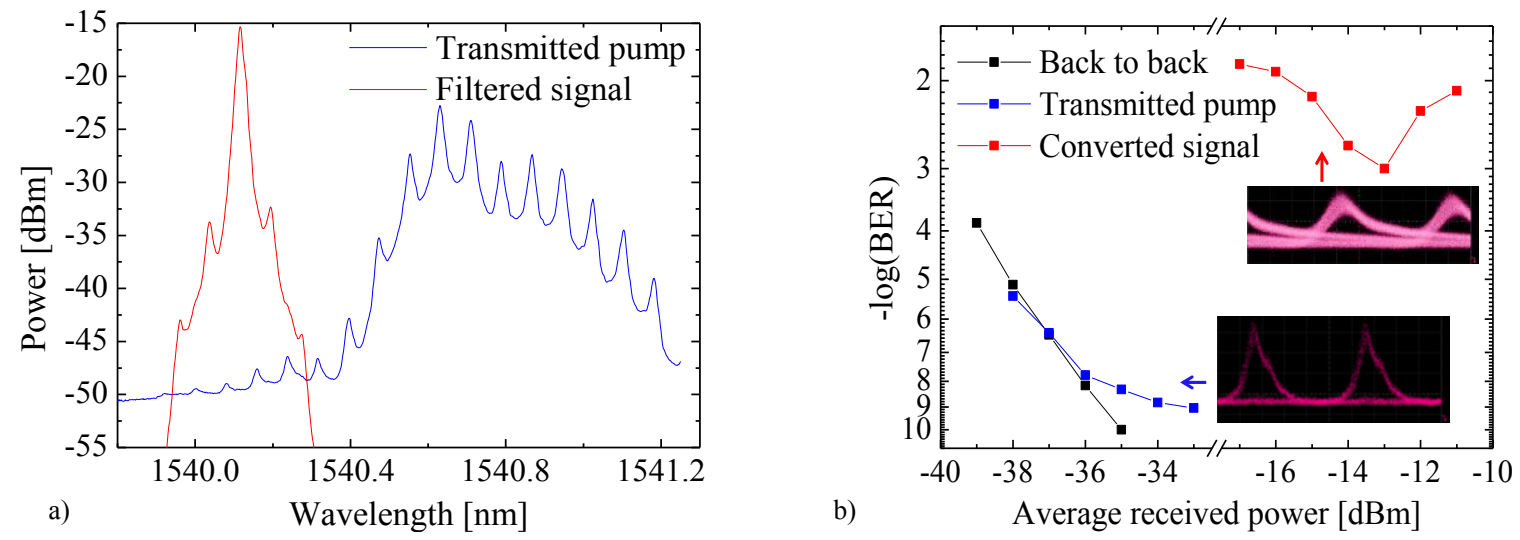

Fig. 3. Transmission spectra of the filtered probe and transmitted pump when the probe is off. b) BER curves of the converted signal, transmitted pump when the signal is off and back-to-back measurement. The insets show the eye diagrams of the converted signal and transmitted pump.

\section{Experimental results}

The spectrum of the converted signal is shown in Fig. 3(a) together with the spectrum of the pump transmitted through the device without the probe. It is clear that the discrete tones observed in the spectrum of the converted signal do not coincide with those of the pump leaking through the cavity and filter. Therefore it indeed means that the probe becomes modulated according to the pump. Having a pump modulated with a PRBS at $10 \mathrm{~Gb} / \mathrm{s}$ with $6.8 \mathrm{dBm}$ average power at the device input and $7.5 \mathrm{~dB}$ of coupling loss leads to a switching energy on the order of $170 \mathrm{fJ}$. The converted signal shows a clear eye diagram, as can be seen in the inset of Fig. 3(b), in spite of the slow 
fall time induced by the slow recombination of the carriers responsible for the cavity shifting, resulting in pulse overlapping and inter-symbol interference. The measured BER exhibits an error floor at $10^{-3}$. In addition to the slow recovery time, in-band crosstalk between the converted signal and part of the pump spectrum leaking through the OBPF affects the BER performance. Nevertheless, the measured BER is below the forward-error-correction (FEC) threshold of $3.3 \times 10^{-3}$ for concatenated codes assuming $7 \%$ overhead, and error free $\left(\mathrm{BER}<10^{-12}\right.$ ) detection is therefore possible at a net rate of $9.35 \mathrm{~Gb} / \mathrm{s}$. The crosstalk issue could be solved by employing a four port device [5] to spatially separate the pump and converted signal. An alternative approach is the use of a device with two resonances separated in frequency [3]. The BER was also measured for the pump transmitted through the cavity without the signal being present. In this case an error floor is observed around a BER of 10 $0^{-9}$, as shown in Fig. 3(b). From the eye diagram of the transmitted pump when the probe is off, also shown as an inset in Fig. 3(b), it can be clearly noticed how the pump signal is distorted due to the limited bandwidth of the cavity.

\section{Conclusion}

Wavelength conversion was successfully demonstrated at $9.35 \mathrm{~Gb} / \mathrm{s}$ using an InP photonic crystal $\mathrm{H} 0$ nanocavity. To the best of our knowledge, this is the first time a switching demonstration is performed with high-speed telecommunication-grade signals and conventional direct detection using such a cavity.

\section{Acknowledgement}

The authors acknowledge financial support from Villum Fonden via the NATEC (NAnophotonics for TErabit Communications) Centre.

\section{References}

[1] K. Nozaki, T. Tanabe, A. Shinya, S. Matsuo, T. Sato, H. Taniyama, and M. Notomi, "Sub-femtojoule all-optical switching using a photoniccrystal nanocavity", Nature Photon. 4, 477-483 (2010).

[2] K. Lenglé, M. Gay, A. Bazin, I. Sagnes, R. Braive, P. Monnier, L. Bramerie, T.-N. Nguyen, C. Pareige, R. Madec, J.-C. Simon, R. Raj, and F. Raineri, "Fast all-optical $10 \mathrm{~Gb} / \mathrm{s}$ NRZ wavelength conversion and power limiting function using hybrid InP on SOI nanocavity", in Proc. ECOC, We.2.E.5., 2012.

[3] S. Combrié, G. Lehoucq, S. Malaguti, G. Bellanca, S. Trillo, J. P. Reithmaier, and A. De Rossi, "Two-color switching and wavelength conversion at $10 \mathrm{GHz}$ using a Photonic Crystal molecule", in Proc. CLEO, CM4D.5., 2013.

[4] Y. Yu, M. Heuck, S. Ek, N. Kuznetsova, K. Yvind, and J. Mørk, "Experimental demonstration of a four-port photonic crystal crosswaveguide structure" Appl. Phys. Lett. 101, 251113 (2012).

[5] Y. Yu, E. Palushani, M. Heuck, S. Ek, N. Kuznetsova, P. Colman, D. Vukovic, C. Peucheret, L. K. Oxenløwe, K. Yvind, and J. Mørk," Low energy, ultra-fast switching using an InP photonic crystal H0 nanocavity" in Proc. CLEO-PR \& OECC/PS, MI1-5, 2013. 